\title{
A Phytochemicals Approach Towards the Treatment of Cervical Cancer Using Polyphenols and Flavonoids
}

\author{
Pooja Yadavi, Ravina Vats', Afsareen Bano', Amit Vashishtha ${ }^{1}$, Rashmi \\ Bhardwaj ${ }^{1 *}$
}

\begin{abstract}
Aim: Despite enormous progress in cancer biology, oncologists are still struggling to retrieve the methods and drugs to cure cancer which remains a global threat to humans. Plant-derived natural compounds, also known as phytochemicals, carry therapeutic potential and could be taken as dietary supplements, which are a radical way to resist as well as cure cancer. The present study reveals the anti-cancerous potential of a few phytochemical constituents under in vitro conditions and their mode of action on cervical cancer. SiHa was treated with phytochemicals viz. Quercetin dihydrate, Gallic Acid, and Naringin with varying concentrations to assess their cytotoxicity potential by various methods and also to elucidate their $\mathrm{IC}_{50}$ values. Methods: All three compounds reduced the cell number and viability, along with alterations in cancer cell morphology. The diagnosed $\mathrm{IC}_{50}$ values of the compounds were $160 \mu \mathrm{M}, 200 \mu \mathrm{M}$, and $1500 \mu \mathrm{M}$ for Quercetin dihydrate, Gallic Acid, and Naringin, respectively. DNA fragmentation assay and gene expression analysis were also used to assess apoptosis and anti-proliferative activity of compounds. Results: We found fragmented DNA in treated cells as assessed by gel electrophoresis assay. These phytochemicals elicit an apoptotic response in SiHa cells by significantly up-regulating the gene expression level of p53 and p21 (p-value $<0.005$ ). Conclusion: Considering the anti-cancer and anti-proliferative potential of Quercetin dihydrate, Gallic Acid, and Naringin on the cervical cancer cell line, these phytochemicals could be used as an alternative or concurrent cancer therapeutic approach. However, further in-depth elucidation of their mode of action, safety, and efficacy should be explored.
\end{abstract}

Keywords: Anticancer activity- cervical cancer- anti-proliferative- phytochemicals- apoptosis- DNA damage

Asian Pac J Cancer Prev, 23 (1), 261-270

\section{Introduction}

Cancer is the major global health burden and cervical cancer is the fourth well-known cancer in terms of mortality and incidence rates (Bray et al., 2018). Although various chemical and physical approaches to treat cancer such as surgery, radiotherapy, chemotherapy, the cancer morbidity rate is still 53\% (Bray et al., 2018). For treatment purposes, various drugs are available in the market. Still, the major pitfall in treating cancer is drug specificity and penetration, along with sensitivity, toxicity, and resistance to existing drugs (Lammers et al., 2012). Thus above issue prompts researchers and clinicians to explore alternative therapies, including natural compounds.

Since ancient times, the uses and importance of natural compounds as a medicine for various ailments have been well established (Dias et al., 2012). Regardless of the multiple application of natural products as medicine, the area still needs exploration. Natural compounds and their native active constituents are widely available naturally and play a significant role as anti-mycotic, antibiotic (Martelli and Giacomini, 2018), anti-cancerous (Grigalius and Petrikaite, 2017), and anti-mitotic (Paier et al., 2018; Kavallaris et al., 2001) agents. Phytochemicals constitute a group of naturally occurring biologically active components such as lipids, phenolics, alkaloids, diterpenes, carotenoids nitrogen-containing compounds (Liu, 2004).

Among phytochemicals, the scientific community has emphasized their interest in flavonoids because of their anti-proliferative property, which serves as a potential therapeutic approach to cancer treatment (Hosseini and Ghorbani, 2015). Flavonoids are polyphenolic compounds derived from secondary metabolites. Based on their biochemical structure, flavonoids are classified into flavanones, flavanols, iso-flavonoids, and anthocyanidins (Panche et al., 2016).

Gallic Acid (GA) represents the primary class of phenolic acid (Dai and Mumper, 2010), while Quercetin dihydrate (QDH) and Naringin (NA) are the foremost 
representatives of the flavanol group of flavonoids (Panche, Diwan and Chandra, 2016), Figure 1. Gallic acid is one of the solitary components of natural resources hence, also known as Ayurvedic herb. Gallic acid is abundantly available in various plant parts such as fruits, seeds, leaves and wood, etc. It is also abundant in edible forms such as blackberry, blueberry, strawberry, cashew nut, walnut, grapes, plums, and mango, and so on (Daglia et al., 2014). QDH is a flavonoid, and some of the best natural food resources of QDH include vegetables and fruits. Vegetables include Onions, Tomatoes, Kale, Broccoli, and peppers, whereas; fruits include Apples, red grapes, cherries, and all berries (blueberry, raspberry, and cranberry) (D'Andrea, 2015). Naringin occurs majorly in citrus fruits, mainly of grapefruits, sour orange, cherries, tomatoes, cocoa, oregano and beans etc. (Alam et al., 2020). Thus, the phytochemicals are found in our diet, including green vegetables, onion, citrus fruit, and green tea (King and Young, 1999; Khan et al., 2020).

GA is one of the widely studied because of its potential role in cancer treatment. GA shows an inhibitory effect on the growth of cancer cells via altering gene expression, which regulates metastasis, angiogenesis, cell cycle, and apoptosis (Zhao and $\mathrm{Hu}, 2013$ ). GA prevents carcinogenesis by stimulating the ATM kinase signaling pathways (Agarwal, Tyagi and Agarwal, 2006). Dose-dependent treatment of GA inhibits cell proliferative activity in human colon, breast, prostate, and oral malignant cell lines (Faried et al., 2007). Quercetin has shown diverse pharmacological activities such as antiviral, anti-cancer, anti-microbial, anti-inflammatory, and anti-obesity agents (David, Arulmoli and Parasuraman, 2016). Hashemzaei et al., (2017), established the inhibitory effect of quercetin on various cancers such as prostate, cervical, lung, breast, and colon while, QDH is less studied and entail to be examined to evaluate its potential role as a drug. Quercetin also has an inductive cytotoxic effect on cancer cells by upregulating the expression of p21 cyclin-dependent kinase inhibitor (Jeong et al., 2009), Bax, caspase-3, and Poly [ADP-ribose] polymerase expression, while downregulating the expression of Bcl2 (Niu et al., 2011).

Moreover, NA is also widely researched, and it has been shown in various studies that NA has bone healing and regeneration capacity (Chen et al., 2016). $750 \mu \mathrm{M}$ concentration of NA showed $50 \%$ inhibition of SiHa human cervical cancer cells via elevating the Bax, caspases, and p53 expression (Ramesh and Alshatwi, 2013). Furthermore, NA modulates the $\beta$-catenin pathway to inhibit the growth potential of breast cancer cells ( $\mathrm{Li}$ et al., 2013). NA inhibits the growth potential of human triple-negative breast cancer cells by targeting the $\beta$-catenin signaling pathway. Given cancer as a threat to humankind and phytochemicals could serve as a novel therapeutic medicine for combating cancer. Our current study aims to study and elucidate the effect of three crucial phytochemical constituents, viz. Gallic acid, Quercetin dihydrate, and Naringin on cervical cancer using cell line model $(\mathrm{SiHa})$. The present study focuses on the molecular mechanism via gene expression alteration and deoxyribonucleic acid (DNA) fragmentation. Also, it depicts morphology alteration in $\mathrm{SiHa}$ cells in a dose- dependent manner for three phytochemicals constituents.

\section{Materials and Methods}

\section{Chemicals, Reagents and plastic ware}

Compounds (GA, QDH, NA from Hi-Media), Trypan blue solution, Dimethyl sulfoxide (DMSO), Fetal bovine serum (FBS), DMEM (Dulbecco's Modified Eagle's Medium), Pen/Strep/Ampho-B, Trypsin/EDTA, Giemsa (HiMedia Pvt. Ltd., India), EDTA (Life Sciences, VWR), Agarose (Lonza), Tris-Acetate-EDTA (GeNeiTM), Ethanol (Merck), Primers (Eurofins), DNA and ribonucleic acid (RNA) ladder (Puregene), Trizol (Ambion), Phenol (VWR), Chloroform (Merck), etc. Plasticware such as cell culture dishes, flasks were procured from Genetix and Tarson.

\section{Cell line}

Cervical Cancer ( $\mathrm{SiHa}$ ) cell line was purchased from NCCS (National Centre for Cell Science, Pune, India). We ensured that cells were free of any contamination before starting the experimental work as depicted microscopically and experiments were done from a young stock (passage 17) as we received them from NCCS at P14.

\section{Stock preparation of compound}

For the stock solution preparation, different compounds were solubilized in different solvents. QDH and NA were soluble in the organic solvent, while Gallic acid was soluble in water. We dissolved $15 \mathrm{mg}$ of GA in one $\mathrm{ml}$ of water to make $79.73 \mathrm{mM}$ of the stock solution; $21 \mathrm{mg} / \mathrm{ml}$ of QDH in ethanol gave $62.0 \mathrm{mM}$ stock solution, and 43.5 $\mathrm{mg} / \mathrm{ml}$ of NA in DMSO gave a stock solution of $75 \mathrm{mM}$

\section{Cell Culture}

The procured SiHa cell line (passage no. 14) from NCCS, Pune, was routinely cultured in a T25 $\mathrm{cm}^{2}$ flask in DMEM. We supplemented the cultured media with $0.1 \%$ Pen/Strep/Ampho-B (10,000UI/100mg/25 $\mu \mathrm{g}$ $-100 \mathrm{X})$ and $10 \% \mathrm{FBS}$. The cultured cells were placed in a $\mathrm{CO}_{2}$ incubator at $37^{\circ} \mathrm{C}$ temperature saturated with $5 \%$ $\mathrm{CO}_{2}$. Cells were trypsinized with trypsin/EDTA solution after attaining 90\% confluency. Before plating, the cell number was determined using trypan blue exclusion assay, and cells were examined over time for bacterial and mycoplasma contamination. The cells were preserved by the cryopreservation method. We used only those vials of cells to assess drug anti-cancer activity, which was passaged at least 6-7 times after thawing but not over 20 passages. SiHa cell line with passage number 18-20 was used for the assessment of compound activity.

\section{Cytotoxicity assay}

a) Trypan Blue exclusion assay: 2 X $10^{5}$ cells $/ 1.5 \mathrm{ml}$ of media were seeded into 6 well plate. After 24 hours, cultured cells were treated with 100, 150, 200, 250, 300, and $350 \mu \mathrm{M}$ of GA, 100, 120, 140, 160, 180 and $200 \mu \mathrm{M}$ of QDH and 1,100, 1,200, 1,300, 1,400, 1,500, and 1,600 $\mu \mathrm{M}$ of NA. After 48 hours of incubation, treated, and untreated (control) cells were trypsinized, pellet down and examined by trypan blue exclusion assay to determine 
cell viability and cell number. The cells were mixed with trypan blue exclusion dye and counted by hemocytometer under the light microscope. Cells were calculated using the formulae given below:

$$
\text { Total cells } / \mathrm{ml}=\text { total no. cell count } / 4 \times \text { dilution factor } \times 10^{4}
$$

b) 3 - ( 4,5-di methy 1 th i a z o $1-2-$ y 1$)-2,5$ diphenyltetrazolium bromide (MTT) Assay: For MTT assay 1 X $10^{4}$ cells were counted on a hemocytometer and seeded into 96 well plate per $0.1 \mathrm{ml}$ of media. After 24 hours, cells were treated with different concentrations of compounds. The cytotoxicity activity of each phytochemical at different concentrations on incubated cells was determined after 48 hours by EZcountTM 3-(4,5-dimethylthiazol-2-yl)-2,5-diphenyl tetrazolium bromide (MTT) cell assay kit (HI Media) as per the manufacturer protocol. To the incubated cells, $10 \mu \mathrm{l}$ of yellow-colored MTT was added, followed by incubation for 3-4 hours in the dark. After incubation, Purple colored formazan developed due to the reduction of MTT by mitochondrial lactate dehydrogenase enzyme of live cells, which were solubilized by adding $100 \mu \mathrm{l}$ of the solubilizing solution to each well. Absorbance was measured at $570 \mathrm{~nm}$ with a reference of $630 \mathrm{~nm}$ of wavelength. The absorbance of untreated cells along with the drugs treated one was used to calculate the $50 \%$ inhibitory concentration $\left(\mathrm{IC}_{50}\right)$ of the drug.

\section{Cell morphology assessments}

a) Giemsa staining: $1.5 \times 10^{5}$ cells in $1 \mathrm{ml}$ of media were seeded into 12 well plate and treated with different concentrations of compounds as discussed above. After 48 hours of incubation, media was removed from treated and untreated (control) cultured cells; cells were fixed by using $1 \mathrm{ml}$ of fixative solution (Ethanol: Chloroform: Glacial acetic acid in 60:30:10 proportion respectively) in each well followed by incubation for 15-20 min. After incubation, the fixative was removed, and cells were stained with Giemsa for 30-40 min and then washed with distilled water. The cell morphology was analyzed by using an inverted microscope.

b)Morphological analysis under Inverted microscope: $\mathrm{SiHa}$ cells treated with different concentrations of compounds along with untreated $\mathrm{SiHa}$ cells were analyzed after $48 \mathrm{hrs}$. of incubation under an inverted microscope (AxioVert 40 CFL, Carl Zeiss) at 10X, 20X, and 40X magnification.

\section{DNA fragmentation analysis}

DNA fragmentation analysis was determined using the gel electrophoresis method. $2 \times 10^{5}$ cells were plated in 6 well plates and treated with $\mathrm{IC}_{50}$ of GA, QDH, and NA. After 48 hours of incubation, the DNA of treated and untreated (control) cells was extracted using a mammalian genomic isolation kit (GeNei) as per the manufacturer's instructions. The quality and quantity of DNA were determined by 260/280 ratio of 1.7-1.9 using nanodrop (Thermo Scientific, USA) and agarose gel electrophoresis. For electrophoresis, isolated DNA was run on $1 \%$ agarose gel and visualized by Gel Doc XR+ system (Bio-Rad).
RNA isolation and Reverse Transcription-Polymerase Chain Reaction (RT-PCR)

To assess drug effect on gene expression levels, RT-PCR was conducted for p21 and p53 genes of treated and untreated (control) cells. The cell line was treated with a $50 \%$ inhibitory concentration of GA, QDH, and NA ( IC $_{50}$ value). After 48 hours of incubation, RNA was isolated using an RNA isolation kit (Qiagen) as per the manufacture protocol. The quantity and quality of isolated RNA were confirmed by a 260/280 ratio of 1.9-2.1 using nanodrop and by agarose gel electrophoresis. Using the First-strand cDNA synthesis kit (Thermo scientific), gene expression of p53 and p21 were examined from isolated RNA using respective primers as given in Table 1. cDNA from RNA was synthesized using 200 ng of RNA. For cDNA synthesis, $20 \mu \mathrm{L}$ of the final volume of reaction mixture consisting of total RNA, oligo (dT) 18 primer, reaction buffer, Ribolock RNase inhibitor, dNTPs, and reverse transcriptase was subjected to RT-PCR cycle as per the manufacture protocol. Final amplification of desired genes was carried out with a PCR Master mix (Pure gene) using $2 \mu \mathrm{l}$ of cDNA synthesized by RT PCR method. Thermal cycling parameters of PCR cycles included initial denaturation at $95^{\circ} \mathrm{C}(3 \mathrm{~min})$, denaturation at $94^{\circ} \mathrm{C}(30 \mathrm{sec})$, annealing at $59.2^{\circ} \mathrm{C}(30 \mathrm{~s}), 54^{\circ} \mathrm{C}(30 \mathrm{~s})$ and $60 \mathrm{oC}(30 \mathrm{~s})$ of $\mathrm{p} 21, \mathrm{p} 53$ and $\beta$ actin (for internal standard reference) respectively followed by 34 PCR cycles with a final extension at $72^{\circ} \mathrm{C}(10 \mathrm{~min})$.

\section{Statistical analysis of data}

All the above-said experiments were performed as triplicates. The data were calculated and represented as the mean \pm standard deviation (SD) of the average value of triplicate observations. Statistical significance between two independent groups was assessed by unpaired student t-test, and one-way ANOVA was used for multiple group comparison. The significant difference between control and compound treated was accepted only after a significant difference in a p-value that is less than 0.05 .

\section{Results}

Natural compounds (GA, QDH, and NA) exhibit cytotoxicity in cervical cancer cell line

Natural compounds have vital capacity to act as anti-proliferative and anti-cancerous agents both in- vivo and in-vitro in various cancers ((Desai et al., 2008). We examined the cytotoxic role of natural compounds on the cervical cell line ( $\mathrm{SiHa}$ ) at different GA, QDH, and NA concentrations in the present study. We assessed the cytotoxicity after $24 \mathrm{hrs}$. and $48 \mathrm{hrs}$. of treatment on logarithmically dividing cells by using trypan blue and MTT assay. Trypan blue exclusion assay revealed that the cytotoxic effect of these compounds was time-dependent, as even after $24 \mathrm{hrs}$. of compounds treatment, the cells retained $\sim 80 \%$ viability which was not significant with respect to control cells, and compounds did not show 50 percent inhibitory concentration $\left(\mathrm{IC}_{50}\right)$ on cancer cells (data not shown). As depicted in Figures 2 and 3 the compounds showed anti-proliferative activity and $\mathrm{IC}_{50}$ after $48 \mathrm{hrs}$.

Asian Pacific Journal of Cancer Prevention, Vol 23 

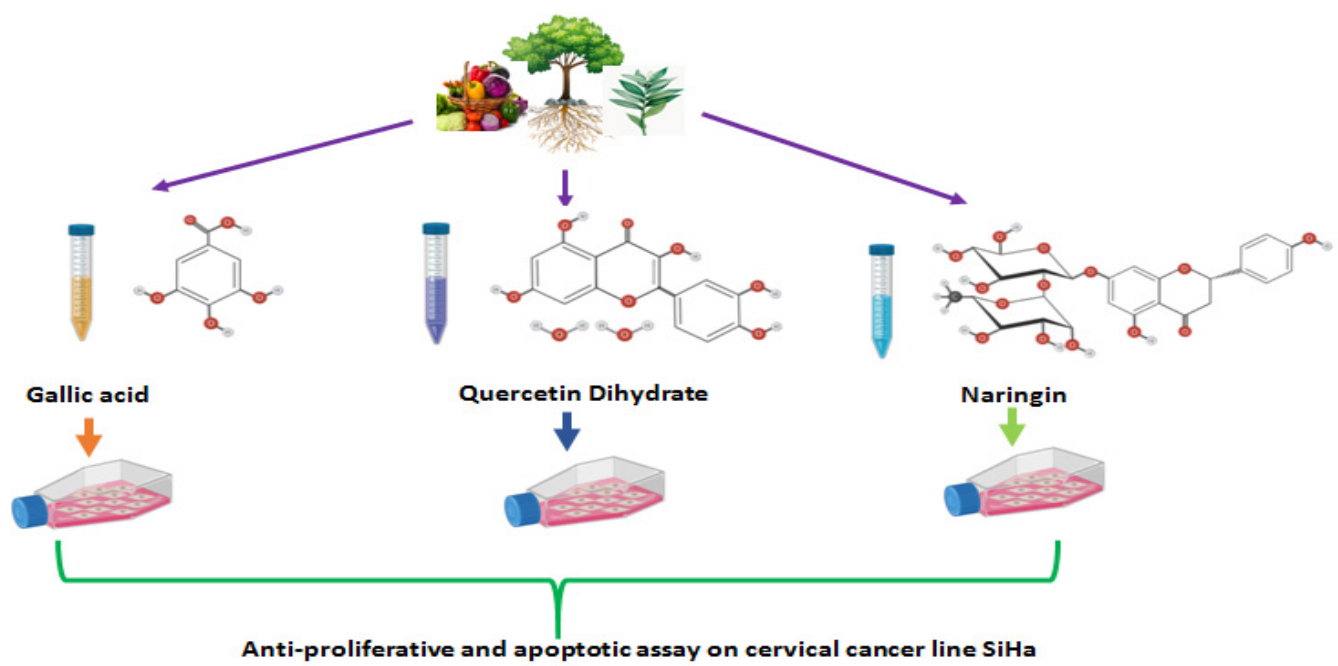

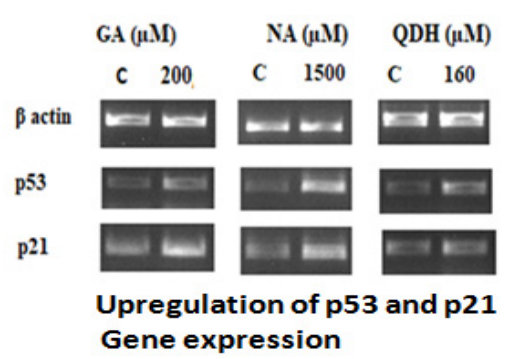

Graphical Abstract of incubation, assayed by trypan blue and MTT assay. As shown in Figure 2(A), 2(B), and 2(C), the calculated IC50 values of GA, QDH, and NA were found to be $200 \mu \mathrm{M}, 160 \mu \mathrm{M}$, and $1500 \mu \mathrm{M}$ respectively on the $\mathrm{SiHa}$ cell line. This formulated that QDH has more putative activity than GA followed by NA. For the assessment of time, the cells were assessed up to $72 \mathrm{hrs}$ (data not shown) after compound treatment, and it was found that increased exposure of compounds beyond $48 \mathrm{hrs}$ leads to severe destruction of cells and thus, we selected $48 \mathrm{hrs}$. for the evaluation of the compound activity. The cell viability and cytotoxic effects of compounds were found to be inversely proportional to time and dose, as shown in Figures 2 and 3 and supplementary Tables 1 and 2 .<smiles>O=C(O)c1cc(O)c(O)c(O)c1</smiles>

Gallic acid<smiles>O=C(O)c1ccc(O)c(O)c1</smiles>

Quercetin dihydrate

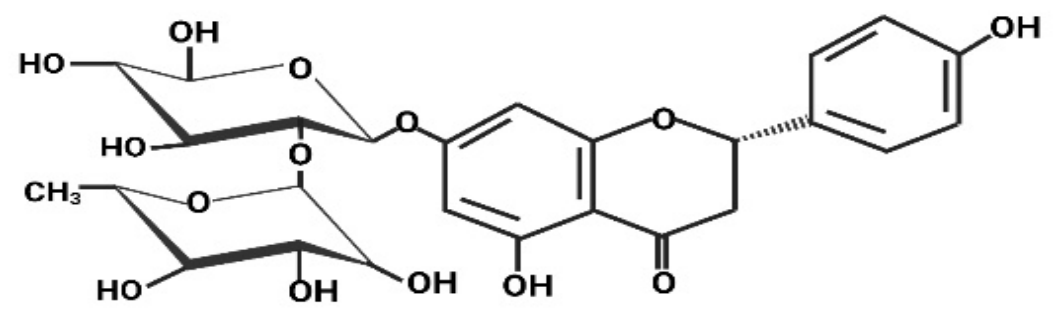

\section{Naringin}

Figure 1. Chemical Structure of Gallic Acid, Quercetin Dihydrate, and Naringin 

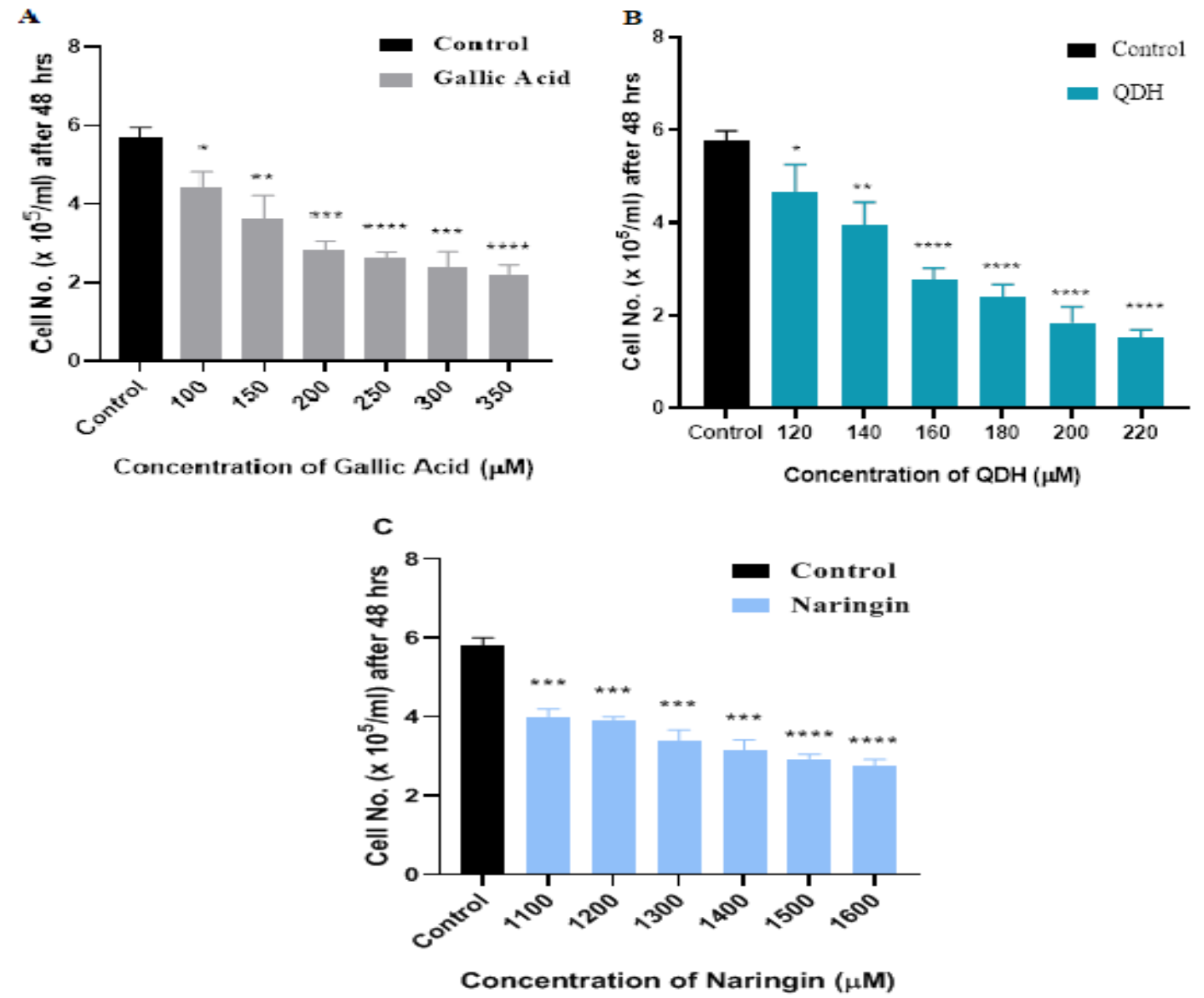

Figure 2. Dose-Response Inhibition Histogram of (A) GA, (B) QDH (C) NA on SiHa after 48hrs of Treatment by Trypan Blue Exclusion Assay. Human cervical cancer cells SiHa were plated in 6 well plates and treated with different concentrations of GA, QDH, and NA, as depicted above. Mean Cell count (x 105 cells $/ \mathrm{ml}$ ) is indicated ( $\mathrm{n}=3$ ) with SD. $\mathrm{IC}_{50}$ of GA, QDH and NA were observed at $200 \mu \mathrm{M}, 160 \mu \mathrm{M}$, and $1500 \mu \mathrm{M}$, respectively.

\section{Altered morphology of cervical cancer cell line}

The GA, QDH, and NA induced morphological changes when studied after $48 \mathrm{hrs}$. of treatment with their respective IC50 values as observed under an inverted microscope at 10X, 20X, and $40 \mathrm{X}$ magnifications. When compared to untreated (control) cells which showed fibroblast shape with a prominent oval-shaped nucleus, treated cells showed the features of apoptotic cells that is cell shrinkage, rounding of cells, loose adherence, and loss of contact with the neighboring cells, as shown in Figure 4. The morphological changes were also observed and confirmed by Giemsa staining, which decreased the extracellular matrix of treated cells, blebbing, nuclear fragmentation, and disintegrated cells. The effect of the above-mentioned natural compounds are concentration- dependent and different compounds tend to depict various morphological changes with respect to control, as shown in Figures 5a, 5b, 5c and 5d (Control, QDH, GA, and NA, respectively). Also, the effect was time-dependent as increased exposure duration of compounds results in enhanced accumulation of debris with more destructed cells.

Natural compounds possess DNA fragmentation potential One of the significant elucidative signs in the process of apoptosis is the fragmentation of chromosomal DNA (Elmore, 2007). To determine the DNA fragmentation activity of natural compounds, we performed the DNA fragmentation assay. First, isolated DNA from treated and control cells after $48 \mathrm{hrs}$ of incubation was evaluated

Table 1. Annealing Temperature and Primer Sequences of p53, p21 and $\beta$ actin Genes for RT-PCR

\begin{tabular}{|c|c|c|c|c|}
\hline Gene & Sequence of primers & Length (bp) & $\mathrm{Tm}$ & Cycle number \\
\hline \multirow[t]{2}{*}{$P 21$} & F: 5'GGAAGGGACACACAAGAAGAA-3' & 300 & $59.2^{\circ} \mathrm{C}$ & 34 \\
\hline & R: 5'-TCCTTGTTCCGCTGCTAATC -3' & & & \\
\hline \multirow[t]{2}{*}{ P53 } & F: 5'GTCTGTGACTTGCACGTACT-3' & 326 & $55^{\circ} \mathrm{C}$ & 34 \\
\hline & R: 5'-CAGTCAGAGCCAACCTCAGG-3' & & & \\
\hline \multirow[t]{2}{*}{$\beta$ actin } & F: 5'-GTGGGGCGCCCCAGGCACCA-3' & 500 & $60^{\circ} \mathrm{C}$ & 34 \\
\hline & R: 5'CTCCTTAATGCACGCACGATTTC-3' & & & \\
\hline
\end{tabular}



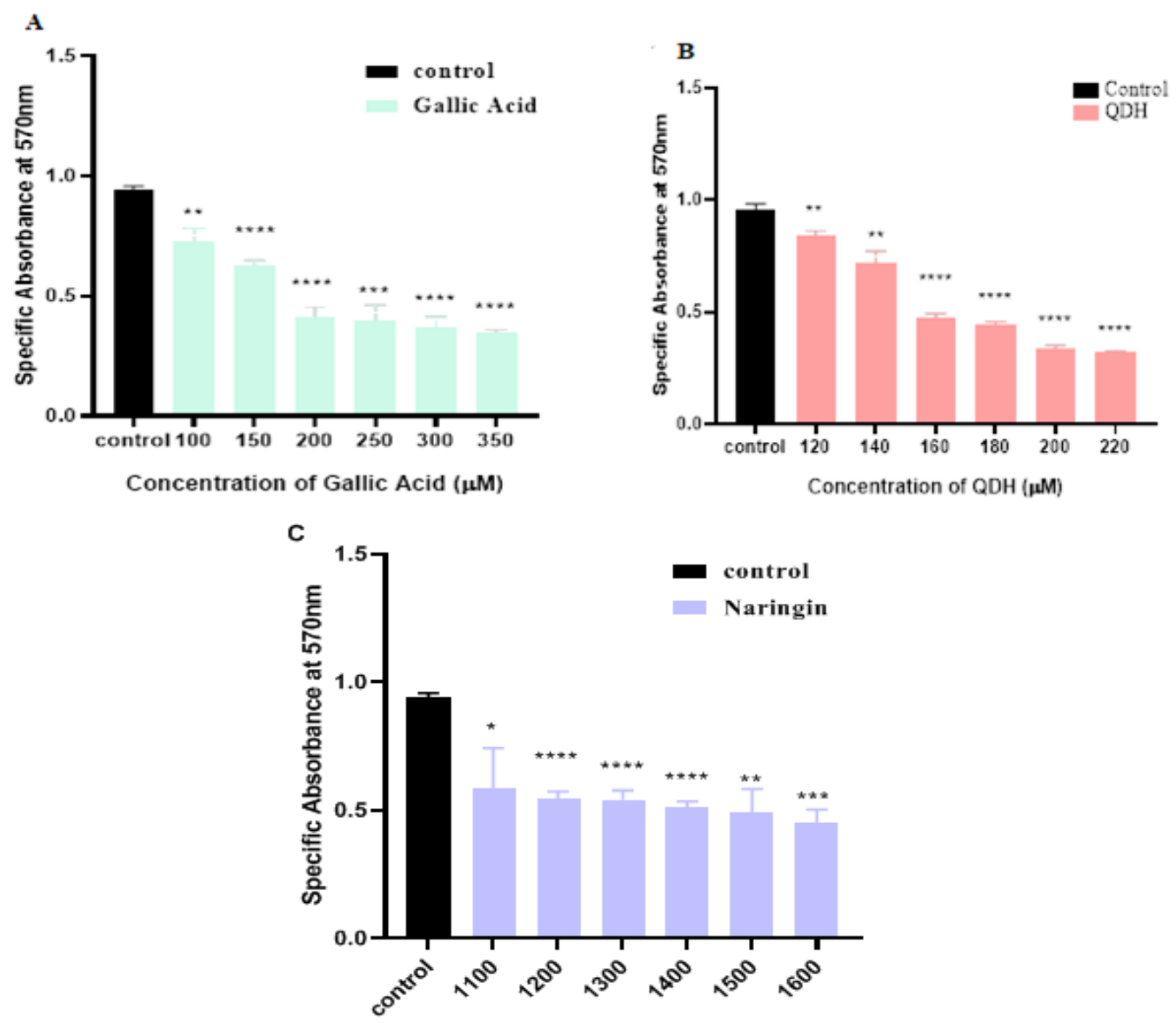

Concentration of Naringin $(\mu \mathrm{M})$

Figure 3. Dose-Response Inhibition Histogram of MTT Assay of Specific Absorbance ( $\lambda=570 \mathrm{~nm})$ with Respect to Concentration of (A) GA, (B) QDH (C) NA on SiHa after 48hrs of Treatment. Mean Cell count (x $104 \mathrm{cells} / \mathrm{ml})$ is indicated $(n=3)$ with SD. IC50 of GA, QDH, and NA were observed at $200 \mu \mathrm{M}, 160 \mu \mathrm{M}$, and $1500 \mu \mathrm{M}$, respectively. A Significant difference with respect to control is represented as $p(<0.05)$ value.

by agarose gel electrophoresis. After electrophoresis, UV visualization of DNA was performed, as shown in Figure 6 confirmed the apoptotic potential with DNA fragmentations by all three phytochemicals.

Effect of phytochemicals on the gene expression level of proteins involved in cell cycle regulator

Our results revealed a significant elevation in p53 and p21 levels in SiHa cells after 48 hours of treatment with GA, NA, and QDH, as shown in Figures 7a, 7b, and 7c, respectively. Outcomes of the present study show that
GA, NA, and QDH target the p21 and p53 genes, both being involved in intrinsic apoptotic signaling pathways and induces cell apoptosis through their up-regulation. Compared to control, treated cells show significant upregulation in $\mathrm{p} 53$ and $\mathrm{p} 21(\mathrm{n}=3$ with $\pm \mathrm{SD})$. The intensity of loaded samples was quantified by Image J software, and the resultant intensity was normalized with beta-actin as an internal control and with non-treated control.
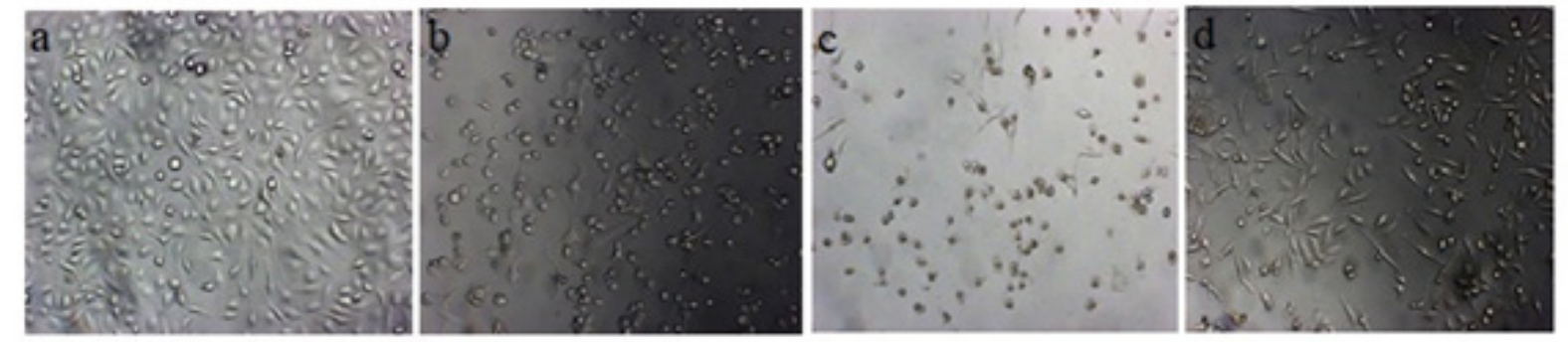

Figure 4. Images of SiHa Cell Line (a) Untreated Cells, (b) QDH, (c) GA, and (d) NA before Giemsa. SiHa cells were cultured in 6 well plate and treated with $\mathrm{IC}_{50}$ of compounds that is $160 \mu \mathrm{M}(\mathrm{QDH}), 200(\mathrm{GA})$ and 1500 (NA). Images were captured by inverted microscope at $10 \mathrm{X}$ after $48 \mathrm{hrs}$. of compound treatment. 


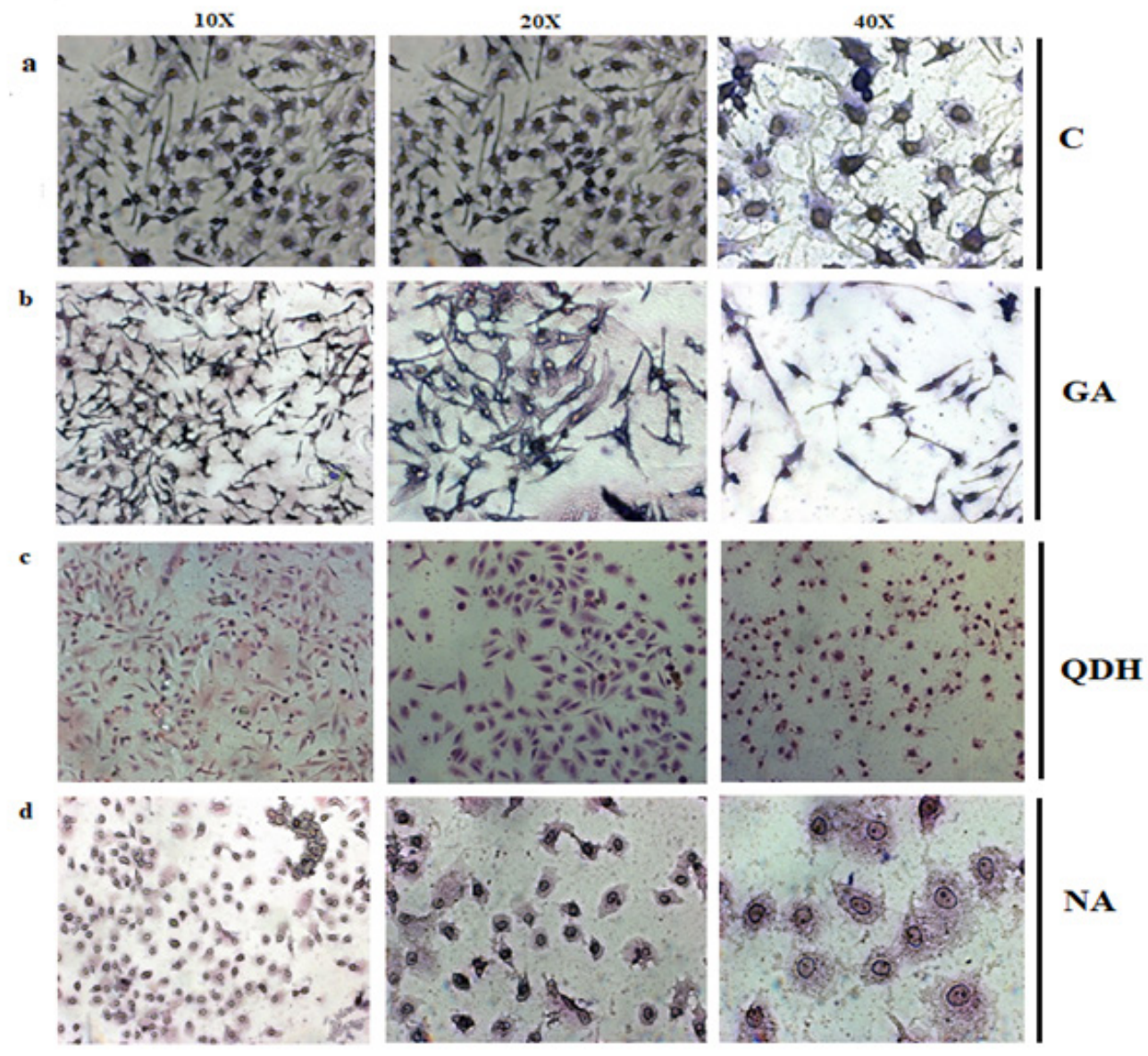

Figure 5. Giemsa Staining of a) Untreated Cells, (b) GA, (c) QDH and (d) NA. SiHa cells were cultured in 6 well plate and treated with IC50 of respective compounds. Images were captured by inverted microscope after $48 \mathrm{hrs}$. of treatment with different magnification that were $10 \mathrm{X}, 20 \mathrm{X}$ and $40 \mathrm{X}$ from left to right.

\section{Discussion}

The alarming incident and mortality rate of cancer is the preeminent threat to the human race (Siegel et al., 2020). Apart from the enormous research prompted in cancer biology by oncologists, cancer persists as the critical reason for mortality. As per the survey by International Agency in Cancer Research (ICAR), cervical

\section{L1 L2 L3 L4 L5}

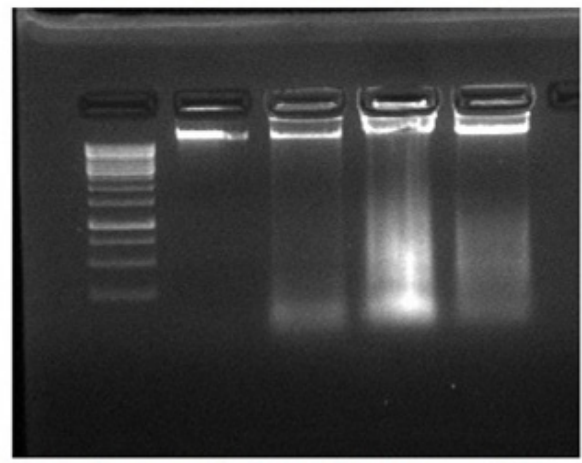

Figure 6. Agarose Gel Electrophoresis Assay for DNA Fragmentation Analysis. DNA of control and compound treated cells were loaded on $1 \%$ agarose gel from left to right. L1: DNA ladder, L2: Control, L3: QDH, L4:GA and L5: NA. cancer is not only the second most abundant cancer type across the globe among women after breast cancer but also it ranks fourth in terms of incidence $(6.6 \%)$ and mortality (7.5\%) rate (Bray et al., 2018). Despite enormous available cancer drugs, the current ones have various pitfalls such as drug resistance and the inability to target cancer stem cells; thus, there is an ardent need to find alternative medicines (Wang et al., 2019)

It is a well-established fact that intake of natural

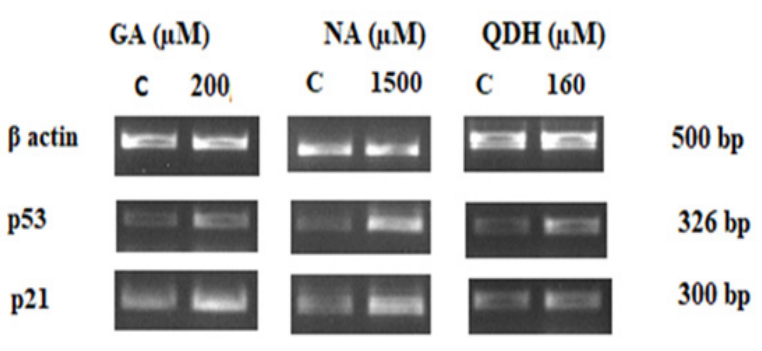

(a)

(b)

(c)

Figure 7. Agar RT-PCR Gene Expression Analysis of p53 and p21 of SiHa after $48 \mathrm{hrs}$ of (a) GA, (b) NA and (c) QDH Treatment. The compound treated and untreated cells (C) were subjected to RT-PCR to evaluate gene expression levels of p53 and p21 with beta actin as an internal control. 
plant products helps promote good health, fight against various disease-causing agents, balance the normal biological process of cells, etc. (Yeum and Russell, 2014). Phytochemicals, the biologically active components found in natural plants, are an emerging tool for cancer treatment as they reduce mortality rates and increase life expectancy (Wang et al., 2012). Most of the in-vivo and in-vitro studies reveal that secondary metabolites such as polyphenols and flavonoids, obtained from polyphenolic plants and fungi, are the widely studied group of phytochemicals that could be exploited as putative drugs for cancer treatment. However, their underlying molecular mechanism by which they elicit their anti-proliferative and apoptotic response to cancer cells is still controversial or unexplained. In the current study, we evaluated the putative anti-cancerous role of three phytochemicals and tried to understand their underlying mechanism of action. The selected three phytochemicals are GA, QDH, and NA. GA belongs to hydroxy-benzoic acid (a subclass of phenolic acid), QDH is a derivative of Quercetin and belongs to flavanol (subclass of flavonoid) while NA represents flavanone (a subclass of flavonoid) group (Basli et al., 2017). In accordance with previous studies done on various cancer cell lines, these phytochemicals do show cytotoxic effects, but their mode of action is still unclear. Moreover, GA and QDH are not yet tested on the SiHa cell line (Meybodi et al., 2017). We have taken these three phytochemicals to evaluate their cytotoxic potential on $\mathrm{SiHa}$ cells through MTT assay, DNA fragmentation assay, cell morphology alteration assay and trypan blue exclusion along with that their underlying mode of action was also assessed via gene expression analysis of cell cycle regulator; p21 and p53.

Oxidoreductase activity of the mitochondrial enzyme of viable cells is used in MTT assay, while trypan blue exclusion assay works on the principle of excluding the dye uptake by viable cells. Both these assays reveal that cytotoxicity of phytochemicals on cancer cells is directly proportional to time and dose; thus, compound exposure time to the cancer cells is a crucial factor in treating cells for $24 \mathrm{hrs}$. does not exhibit significant results as compared to control cells (Supplement Table 2). Furthermore, MTT and trypan assay results concluded that these phytochemicals reduce cell viability by interrupting the normal metabolism of mitochondria and disorganizing the cell structure, which is concurrent with the already present study.

Phytochemicals GA, QDH, and NA depicted different 50 percent inhibitory concentrations $\left(\mathrm{IC}_{50}\right.$ values $)$ on cervical cancer cell line because of their different biochemical structure (Naringin $\mid \mathrm{C}_{27} \mathrm{H}_{32} \mathrm{O}_{14}$ - PubChem, no date; Quercetin dihydrate $\mid \mathrm{C}_{15} \mathrm{H}_{14} \mathrm{O}_{9}$ - PubChem, no date; PubChem, 2011). After 48 hrs. of compound treatment on cancer cells, $\mathrm{IC}_{50}$ values of phytochemicals were $160 \mu \mathrm{M}$, $200 \mu \mathrm{M}$, and $1,500 \mu \mathrm{M}$ for QDH, GA, and NA, respectively (Figures 2 and 3). The IC50 value concluded that QDH is a more effective anti-cancerous agent followed by GA and NA.

Phytochemicals also act as apoptotic agents and alter the morphology of cancer cells (Abotaleb et al., 2019). Cancer cells, once treated with phytochemicals, illustrate the hallmark sign of apoptosis, which are blebbing, reduced cytoplasm, fragmented cells, while control cells exhibit their ability to adhere, maintain their fibroblast shape, intact nucleus, and plasma membrane. Our experimental results of morphological alteration of cells when treated with GA, QDH and NA are analogous to research done by other researchers on various other different cell lines (Zeng et al., 2014; Xie et al., 2017). DNA of treated and control cells confirms the apoptotic effect of phytochemicals as depicted by gel electrophoresis assay. The critical regulatory feature is its balanced cell division maintained by various checkpoints such as DNA damage checkpoints and spindle assembly checkpoints (Kastan and Bartek, 2004). p53 is a tumor suppressor gene that halts the systematic progression of the cell cycle from the $\mathrm{S}$ phase to the $\mathrm{M}$ phase. The p53 induced apoptosis pathways are activated by damaged DNA, lack of growth factors, or hypoxia-induced damage (Haupt et al., 2003). p21 works reciprocally with p53 gene-induced growth inhibition (Kastan and Bartek, 2004). The present observation of experiments showed a significant alteration in the gene expression pattern of p21 and p53 when $\mathrm{SiHa}$ cells were treated for $48 \mathrm{hrs}$. with $\mathrm{IC}_{50}$ of different phytochemicals. The experimental results concluded that modulation of gene expression of $\mathrm{p} 21$ is directly related to p53 in treated cells compared to untreated cells, affirming their coupled association in cell cycle regulation. There is a direct link between the up-regulation of tumor suppressor gene p53 and cell growth reduction along with the decrease in cell number. The upregulation of p21 and p53 in our study coincides with several other previously done studies. Numerous studies show that treating different cancer cell line with GA manifest increase in expression levels of p21 and p53 genes resulting in cell apoptosis, morphological alteration, anti-angiogenesis and DNA damage (Zhao and $\mathrm{Hu}, 2013$; He et al., 2016). The anti-cancerous and gene modulation activity of Quercetin is also well established. Quercetin induces apoptosis in bladder cancer cells and also shows an inhibitory effect on the growth of cancerous cells by decreasing the expression of survivin proteins (Ma et al., 2006). It has been established by researchers that dose-dependent usage of QA is not only effective in persuading apoptosis of cancer cells via enhanced expression of p53 and p21 (Mu et al., 2007) but also it activates the AMPK pathway (Su et al., 2016), reduces cancer cell proliferation significantly (Yang et al., 2006), exhibits DNA fragmentation and anti-angiogenesis potential (Lu et al., 2018). Apart from the intense research done on Quercetin, there is still a need for elucidating the anti-cancerous effect of QDH. Various studies showed that NA treatment inhibits the proliferation of different cancer cell lines through significant up-regulation of p53, Bax, and p21 (Ramesh and Alshatwi, 2013; Cheng et al., 2020). In a study performed by Erdogan et al., (2018), NA significantly upregulated the expressions of p53 and p21 in prostate cancer cell line along with downregulation of survivin protein. Our study of Naringin is concordant with the results depicted by Ramesh et al., (2013) in which NA showed DNA fragmentation, cell death, and morphological alteration on $\mathrm{SiHa}$ cell line (Data from our study showed that the above-selected compounds significantly alters cells morphology, reduce cell viability, 
induce DNA fragmentation and upregulate the expression of p53 and p21 genes in SiHa cells indicating their importance in increasing life expectancy of cervical cancer patients. Our results illustrate that the gene expression of p53 and p21 in SiHa was modulated when treated with phytochemicals; however, the molecular mechanism of a cell is a bit complex; also the elucidation of one mode of action is not sufficient to explicate the mechanistic pathway of phytochemical-induced anti-proliferative and apoptosis potential on cancer cells.

In conclusion, as illustrated by our results and many other researchers, natural products and their derivative compounds (or secondary metabolites) may serve as a promising approach for potential anti-cancer drug discovery. Our current study, conducted on cervical cancer cell line with GA, QDH, and NA suggested that the phytochemicals present in natural compounds induce cytotoxic effects on cancer cells via apoptosis and inhibiting proliferation. Our findings propose that GA, QDH, and NA are potential candidates that can be used as therapeutic agents and helpful in increasing cancer survival rate, decreasing mortality and health burden caused due to cancer. Therefore, the dietary foods rich in the above-mentioned phytochemicals can act as a potent factor to impede, prevent, and cure cancer. Also, it is worth recognizing the fact that further investigation needs to be done in this field to conclude that these phytochemicals are the targeted inhibiting agents for cancer. Experimenting with comparing phytochemicals on different cancer cell lines and evaluating them in in-vivo models would enhance the better understanding of phytochemical's role as a therapeutic agent.

\section{Abbreviations}

3-(4,5-dimethylthiazol-2-yl)-2,5-diphenyl tetrazolium bromide

DNA: Deoxyribonucleic acid

DMSO: Dimethyl sulfoxide

DMEM: Dulbecco's Modified Eagle's Medium

FBS: Fetal bovine serum

GA: Gallic Acid

$\mathrm{IC}_{50}: 50 \%$ inhibitory concentration

NA: Naringin

NCCS: National Centre for Cell Science

QDH: Quercetin dihydrate

RNA: Ribonucleic acid

RT-PCR : Reverse Transcription-Polymérase Chain Réaction

$\mathrm{SD}:$ Standard déviation

\section{Author Contribution Statement}

PY and RV set up the experiments, analyzed and interpreted the data, collected literature, drafted, designed, wrote and edited the manuscript. AB and AV analyzed, interpreted the data and edited the manuscript. RB conceptualized the outline, topic of the article, designed the study and edited the manuscript for submission. All authors read and approved the final manuscript. All authors read and approve the manuscript for submission.

\section{Acknowledgments}

Authours are greatful to Centre for Medical Biotechnology, Maharshi Dayanand University, Rohtak and Council of Scientific \& Industrial Research (CSIR), Pusa New Delhi for providing financial support to accomplish this work. This study is not part of an approved student thesis. Since the research work has been done on preestablished cell line and no fresh clinical samples has been used, also, no clinical data has been generated through this research, no ethical issues is associated with this research. The authors declare that they have no conflict of interest.

\section{Availability of data and materials}

All data generated or analyzed during this study are included in this published article.

\section{References}

Agarwal C, Tyagi A, Agarwal R (2006). Gallic acid causes inactivating phosphorylation of $\operatorname{cdc} 25 \mathrm{~A} / \mathrm{cdc} 25 \mathrm{C}-\mathrm{cdc} 2$ via ATM-Chk2 activation, leading to cell cycle arrest, and induces apoptosis in human prostate carcinoma DU145 cells. Mol Cancer Ther, 5, 3294-302.

Alam F, Kharya AK, Juber A, et al (2020). Naringin: Sources, Chemistry, Toxicity, Pharmacokinetics, Pharmacological Evidences, Molecular Docking and Cell line Study. Res $J$ Pharm Technol, 13, 2507-15.

Basli A, Belkacem N, Amrani I (2017). Health benefits of phenolic compounds against cancers, London, UK, IntechOpen.

Bray F, Ferlay J, Soerjomataram I, et al (2018). Global cancer statistics 2018: GLOBOCAN estimates of incidence and mortality worldwide for 36 cancers in 185 countries. $C A$ Cancer J Clin, 68, 394-424.

Chen R, Qi Q-L, Wang M-T, et al (2016). Therapeutic potential of naringin: an overview. Pharm Biol, 54, 3203-10.

Cheng H, Jiang X, Zhang Q, et al (2020). Naringin inhibits colorectal cancer cell growth by repressing the PI3K/AKT/ mTOR signaling pathway. Exp Ther Med, 19, 3798-804.

D'Andrea G (2015). Quercetin: a flavonol with multifaceted therapeutic applications?. Fitoterapia, 106, 256-71.

Daglia M, Di Lorenzo A, F Nabavi S, et al (2014). Polyphenols: well beyond the antioxidant capacity: gallic acid and related compounds as neuroprotective agents: you are what you eat!. Curr Pharm Biotechnol, 15, 362-72.

Dai J, Mumper RJ (2010). Plant phenolics: extraction, analysis and their antioxidant and anticancer properties. Molecules, 15, 7313-52.

David AVA, Arulmoli R, Parasuraman S (2016). Overviews of biological importance of quercetin: A bioactive flavonoid. Pharm Rev, 10, 84.

Desai AG, Qazi GN, Ganju RK, et al (2008). Medicinal plants and cancer chemoprevention. Curr Drug Metabolism, 9, 581-91.

Dias DA, Urban S, Roessner U (2012). A historical overview of natural products in drug discovery. Metabolites, 2, 303-36.

Elmore S (2007). Apoptosis: a review of programmed cell death. Toxicol Pathol, 35, 495-516.

Erdogan S, Doganlar O, Doganlar ZB, et al (2018). Naringin sensitizes human prostate cancer cells to paclitaxel therapy. Prostate Int, 6, 126-35.

Faried A, Kurnia D, Faried L, et al (2007). Anticancer effects of gallic acid isolated from Indonesian herbal medicine,

Asian Pacific Journal of Cancer Prevention, Vol 23 
Phaleria macrocarpa (Scheff.) Boerl, on human cancer cell lines. Int J Oncol, 30, 605-13.

Grigalius I, Petrikaite V (2017). Relationship between antioxidant and anticancer activity of trihydroxyflavones. Molecules, 22, 2169.

Hashemzaei M, Delarami Far A, Yari A, et al (2017). Anticancer and apoptosis-inducing effects of quercetin in vitro and in vivo. Oncol Rep, 38, 819-28.

Haupt S, Berger M, Goldberg Z, et al (2003). Apoptosis-the p53 network. J Cell Sci, 116, 4077-85.

He Z, Chen AY, Rojanasakul Y, et al (2016). Gallic acid, a phenolic compound, exerts anti-angiogenic effects via the PTEN/AKT/HIF-1 $\alpha /$ VEGF signaling pathway in ovarian cancer cells. Oncol Rep, 35, 291-7.

Hosseini A, Ghorbani A (2015). Cancer therapy with phytochemicals: evidence from clinical studies. Avicenna $J$ Phytomed, 5, 84.

Jeong JH, An JY, Kwon YT, et al (2009). Effects of low dose quercetin: Cancer cell specific inhibition of cell cycle progression. J Cell Biochem, 106, 73-82.

Kastan MB, Bartek J (2004). Cell-cycle checkpoints and cancer. Nature, 432, 316-23.

Kavallaris M, Verrills NM, Hill BT (2001). Anticancer therapy with novel tubulin-interacting drugs. Drug Resistance Updates, 4, 392-401.

Khan T, Ali M, Khan A, et al (2020). Anticancer plants: A review of the active phytochemicals, applications in animal models, and regulatory aspects. Biomolecules, 10, 47.

King A, Young G (1999). Characteristics and occurrence of phenolic phytochemicals. J Am Dietetic Association, 99, 213-8.

Lammers T, Kiessling F, Hennink WE, et al (2012). Drug targeting to tumors: principles, pitfalls and (pre-) clinical progress. J Controlled Release, 161, 175-87.

Li H, Yang B, Huang J, et al (2013). Naringin inhibits growth potential of human triple-negative breast cancer cells by targeting $\beta$-catenin signaling pathway. Toxicol Lett, 220, 219-28.

Liu RH (2004). Potential synergy of phytochemicals in cancer prevention: mechanism of action. $J$ Nutr, 134, 3479S-85S.

Lu J, Wang Z, Li S, et al (2018). Quercetin inhibits the migration and invasion of HCCLM3 cells by suppressing the expression of p-Akt1, matrix metalloproteinase (MMP) MMP-2, and MMP-9. Int Med J Exp Clin Res, 24, 2583.

Ma L, Feugang JM, Konarski P, et al (2006). Growth inhibitory effects of quercetin on bladder cancer cell. Front Bioscience, 11, 2275-85.

Martelli G, Giacomini D (2018). Antibacterial and antioxidant activities for natural and synthetic dual-active compounds. Eur J Med Chem, 158, 91-105.

Mollakhalili Meybodi N, Mortazavian AM, Bahadori Monfared A, et al (2017). Phytochemicals in cancer prevention: a review of the evidence. Iran J Cancer Prev, 10.

Mu C, Jia P, Yan Z, et al (2007). Quercetin induces cell cycle G1 arrest through elevating Cdk inhibitors p21 and p27 in human hepatoma cell line (HepG2). Methods Findings Exp Clin Pharmacol, 29, 179-84.

Naringin |C27H32O14 - PubChem (no date). Available at:https:// pubchem.ncbi.nlm.nih.gov/compound/naringin.

Niu G, Yin S, Xie S, et al (2011). Quercetin induces apoptosis by activating caspase-3 and regulating Bcl-2 and cyclooxygenase-2 pathways in human HL-60 cells. Acta Biochimica et BiophysicaSinica, 43, 30-7.

Paier CRK, Maranhão SSA, Carneiro TR, et al (2018). Natural products as new antimitotic compounds for anticancer drug development. Clinics, $\mathbf{7 3}$.

Panche AN, Diwan AD, Chandra SR (2016). Flavonoids: an overview. J Nutr Sci, 5.

PubChem (2011) 'Gallic acid - Compound Summary', Compound Summary, 5936077, pp 1-36. doi: 10.5517/ ccw76q0.

Quercetindihydrate $\mid \mathrm{C} 15 \mathrm{H} 14$

Wang X, Zhang H, Chen X (2019). Drug resistance and combating drug resistance in cancer. Cancer Drug Resistance, 2, 141-60.

Xie D, Yuan P, Wang D, et al (2017). Effects of naringin on the expression of miR-19b and cell apoptosis in human hepatocellular carcinoma. Oncol Lett, 14, 1455-9.

Yang J-H, Hsia T-C, Kuo H-M, et al (2006). Inhibition of lung cancer cell growth by quercetin glucuronides via G2/M arrest and induction of apoptosis. Drug Metab Dispos, 34, 296-304.

Yeum K-J, Russell RM (2014). Biological Functions of Plant Pigment Phytochemicals in Humans.In 'Systems Biology of Free Radicals and Antioxidants', Eds Springer Berlin Heidelberg, Berlin, Heidelberg, pp 4023-45.

Zeng L, Zhen Y, Chen Y, et al (2014). Naringin inhibits growth and induces apoptosis by a mechanism dependent on reduced activation of NF-kB/COX-2-caspase-1 pathway in HeLa cervical cancer cells. Int J Oncol, 45, 1929-36.

Zhao B, Hu M (2013). Gallic acid reduces cell viability, proliferation, invasion and angiogenesis in human cervical cancer cells. Oncol Lett, 6, 1749-55.

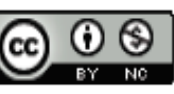

This work is licensed under a Creative Commons AttributionNon Commercial 4.0 International License. 